\title{
Implications des choix alimentaires des ovins et des bovins dans les parcours à Teline linifolia pour l'aménagement de la subéraie de la Mâamora (Maroc)
}

\author{
Ahmed Hracherrass ${ }^{1}$ \\ Omar Berkat ${ }^{2}$ \\ François-Xavier De Montard ${ }^{3}$ \\ ${ }^{1}$ Département de foresterie \\ et des ressources naturelles \\ Institut agronomique et vétérinaire (IAV) \\ Hassan-II \\ BP 6202 Rabat-Instituts \\ 10101 Rabat \\ Maroc \\ <hracherrass2003@yahoo.fr> \\ 2 Département d'écologie et de pastoralisme \\ IAV Hassan-II \\ BP 6202 Rabat-Instituts \\ 10101 Rabat \\ Maroc \\ <berkatomar@yahoo.fr> \\ ${ }^{3} 63111$ Dallet \\ France \\ $<$ fdemontard@free.fr>
}

\begin{abstract}
Résumé
Depuis un siècle, la forêt de chêne-liège de la Mâamora (Maroc) a beaucoup souffert d'une pression pastorale croissante. Elle abrite des peuplements denses de Teline linifolia, une légumineuse arbustive vigoureuse qui pourrait contribuer à la régénération des sols et de la forêt à condition de constituer aussi une ressource pastorale conséquente. Des mesures mensuelles in situ sur un an montrent qu'en automne la faiblesse de l'offre d'herbe et d'organes verts arbustifs est en partie compensée par le renouveau précoce du feuillage de $T$. linifolia contribuant jusqu'à 24 et $12 \%$ de la vitesse globale d'ingestion des ovins et des bovins respectivement. La place que la culture de Teline pourrait tenir dans la politique de régénération et de multi-usage de la subéraie est mise en lumière et discutée.
\end{abstract}

Mots clés : bovins ; chêne-liège ; Maroc ; ovins ; préférence alimentaire ; régénération ; système sylvopastoral.

Thèmes : productions animales ; productions végétales ; ressources naturelles et environnement.

\begin{abstract}
Implications of sheep and cattle Teline linifolia bush ingestion behaviour for managing the Maamora cork tree forest (Morocco)

The cork tree forest of Maamora near Rabat (Maroc) has suffered deep and increasing grazing pressure for 100 years. Dense understorey stands of Teline linifolia, a native, nitrogen fixing and vigorous legume shrub, would contribute to forest regeneration provided that it be proven to be a resource for sheep and cattle grazing. Autumn is a period with lower resources of grass and with no nitrogen fixing shrubs. In situ monthly measurements of sheep and cattle ingestion rates show a significant contribution of T. linifolia in both sheep (up to 24\%) and cattle (up to 12\%) total ingestion rates in autumn, due to its peculiar phenology along with the inadequacy of grass and other shrub resources at this time. The opportunity of planting Teline shrubs in the process of cork tree forest regeneration along with multi-purpose uses is described and discussed.
\end{abstract}

Key words: cattle; cork oak; feeding preferences; Morocco; regeneration; sheep; silvopastoral systems.

Subjects: animal productions; natural resources and environment; vegetal productions.

A ux portes de Rabat, cernée par une population de trois millions d'habitants dont 300000 ruraux, la forêt naturelle de chêne-liège (subéraie) de la Mâamora (figure 1) répond à des usages multiples : loisirs, productions de liège, charbon de bois, bois de feu, champignons, plantes aromatiques et médicinales, miel, glands, rameaux d'émondage et pâturage en sous-bois. La Mâamora est un plateau sableux situé entre 10 et $280 \mathrm{~m}$ d'altitude à l'étage ther- 
La subéraie en forêt de la Maâmora

(in Fraval et Villemant, 1997)

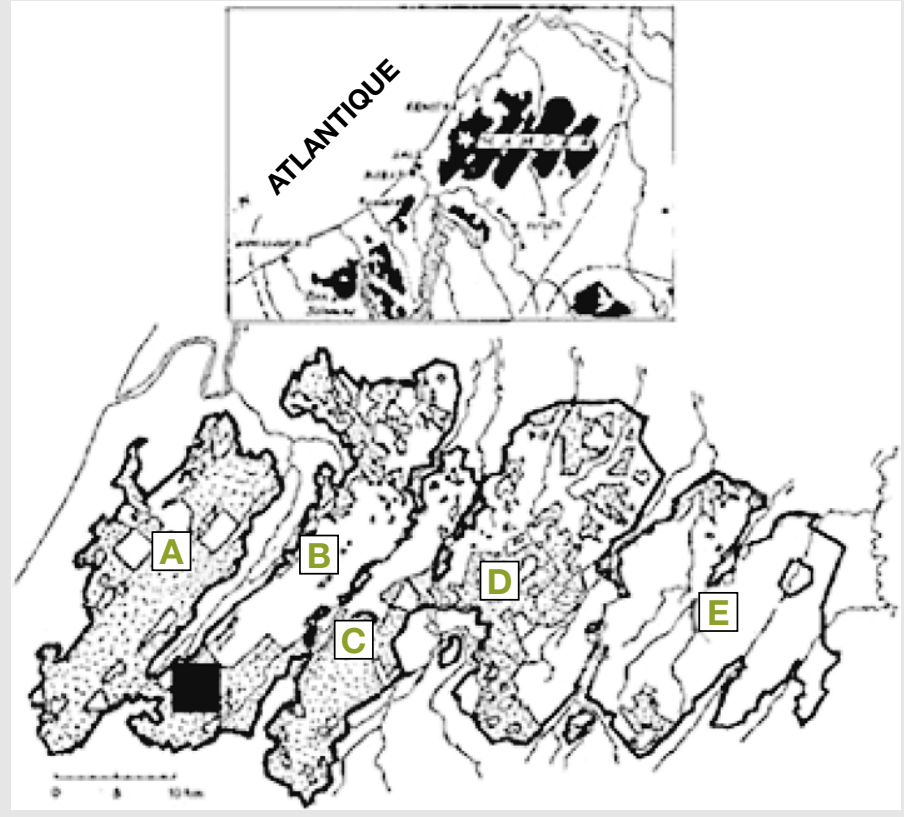

A, B , C, D, E : les cinq cantons de la Maàmora ; zone d'étude; en grisé : la subéraie ; en blanc : les plantations d'eucalyptus et de pins

Figure 1. La forêt de la Mâamora, situation, limites et occupation du sol.

Figure 1. Maamora forest: position, limits and land management.

moméditerranéen, subhumide à hiver chaud à l'ouest $(p=600 \mathrm{~mm})$ et semiaride à hiver tempéré au centre et à l'est ( $p=450 \mathrm{~mm})$. D'après Aafi (2007), la végétation appartient à la série thermoméditerranéenne du chêne-liège dégra- dée par une très forte pression anthropique (tableau 1).

Au début du $\mathrm{xx}^{\mathrm{e}}$ siècle, la subéraie couvrait 135000 ha avant que s'y exerce une pression pastorale croissante et très intense (tableau 2) ; (Iraki et al., 1991 ;
M'Hirit et al., 1995 ; Qarro, 1995 ; Nafaa, 1997). L'augmentation de la population rurale périphérique et les mises en pension d'animaux par les citadins ont fortement accru l'effectif des herbivores (Iraki et al., 1991 ; Berkat, 1995) et considérablement dégradé la forêt. La récolte des glands doux, le broutage des jeunes semis, l'émondage des arbres entraînent un très faible renouvellement et une forte mortalité des arbres, en majorité issus de recépages, peu vigoureux et très sensibles aux attaques des insectes (Fraval et Villemant, 1997).

Face à une évolution destructive, les forestiers ont remplacé les chênes-lièges défaillants par des plantations d'eucalyptus, de pins et d'acacia pour l'industrie (pâte à papier, bois d'œuvre, tannins) ; la stérilité de leur sous-bois a entraîné l'accroissement de la pression pastorale dans la subéraie au détriment des mises en défens de régénération. Ces plantations exotiques répondent mal aux exigences d'un aménagement durable et à la demande sociale d'un meilleur aménagement touristique et sylvopastoral.

Les populations urbaine et rurale du Maroc ont été multipliées par 4,1 et 3,4\% respectivement entre 1960 et 2004 : la mise en cohérence des différents usages de la forêt, écotourisme, sylvopastoralisme et exploitation des produits forestiers, est activement recherchée (Benzyane et al., 2002 ; Centre de recherche forestière, 2008). Dans cette perspective, une meilleure connaissance du comportement alimentaire des ovins et des bovins dans le sous-bois restait

\section{Tableau 1. Groupements végétaux de la subéraie de la Mâamora (d'après Aafi, 2007).}

Table 1. Vegetation groups in the Maamora forest (from Aafi, 2007).

\begin{tabular}{|c|c|c|c|c|}
\hline $\begin{array}{l}\text { Série } \\
\text { thermoméditerranéenne } \\
\text { à chêne-liège }\end{array}$ & & Cantons & $\begin{array}{l}\text { Surface } \\
\text { en } \\
\text { hectares }\end{array}$ & $\begin{array}{l}\text { Densité } \\
\text { (nombre de tiges } \\
\text { par hectare) }\end{array}$ \\
\hline $\begin{array}{l}\text { Quercus suber et } \\
\text { Teline linifolia } \\
\rightarrow\end{array}$ & $\begin{array}{l}\text { Climax de l'Ouest } \\
\text { et du Centre }\end{array}$ & $\begin{array}{l}\text { Plages en } A, B \text { et } D \text { sud et } \\
\text { dans les mises en défens }\end{array}$ & 3151 & Forte $>200$ \\
\hline $\begin{array}{l}\text { - Sous association } \\
\rightarrow\end{array}$ & $\begin{array}{l}\text { À Thymelea lythroïdes } \\
\text { (mattoral) dégradation } \\
\text { réversible }\end{array}$ & $\begin{array}{l}\text { A, B nord zone occidentale } \\
\text { humide }\end{array}$ & 22495 & $\begin{array}{l}\text { Moyenne } \\
100 \text { à } 200\end{array}$ \\
\hline $\begin{array}{l}\text { - Sous association } \\
\rightarrow\end{array}$ & $\begin{array}{l}\text { À Chamaerops humilis } \\
\text { (mattoral) forte dégradation }\end{array}$ & $\begin{array}{l}\text { D, B sud, C sud, sables sur } \\
\text { plancher argileux peu profond }\end{array}$ & 26682 & Faible $<100$ \\
\hline $\begin{array}{l}\text { - Sous association } \\
\rightarrow\end{array}$ & $\begin{array}{l}\text { À Cistus salviifolius (mattoral) } \\
\text { dégradation prononcée }\end{array}$ & A sud, $C$ sud & 3046 & Faible à moyenne \\
\hline $\begin{array}{l}\text { Halimium halimifolium et } \\
\text { Cistus crispus }\end{array}$ & Climax de I'Est & E zone orientale sèche & 3022 & Faible $<100$ \\
\hline $\begin{array}{l}\text { Malcomia triloba et } \\
\text { Urginea maritima }\end{array}$ & $\begin{array}{l}\text { Pelouses sans arbres } \\
\text { Dégradation très poussée }\end{array}$ & $\begin{array}{l}\text { Clairières et zones vides } \\
\text { d'arbres }\end{array}$ & 7350 & Nulle \\
\hline
\end{tabular}


à acquérir (Mejjati Alami, 1991 ; Hracherrass, 2004).

\section{Une question non résolue : le rôle pastoral de Teline}

Une composante importante du sousbois, l'arbuste Teline linifolia (L) Webb et Bert est une légumineuse calcifuge, de 1 à $3 \mathrm{~m}$ de hauteur, à feuillage persistant. Sa bonne adaptation sous couvert de chêne-liège, son rôle protecteur vis-à-vis des jeunes plants, les remontées biologiques résultant de son large enracinement et son aptitude à fixer l'azote de l'air, pourraient faciliter la régénération de la fertilité du sol et le redéploiement du chêne-liège.

Dans la perspective de l'aménagement sylvopastoral, il est nécessaire de connầtre dans quelle mesure cet arbuste peut participer à l'alimentation des troupeaux dès lors que les arbres deviendront défensables. Une expérimentation a été menée pour mettre en évidence l'intérêt pastoral de T. linifolia au cours d'un cycle annuel. Son rôle potentiel dans la régénération de la subéraie et de sa ressource pastorale sera discuté dans la perspective d'une réorganisation du multi-usage et d'une prise de responsabilité des usagers.

\section{Matériel et méthode}

L'objectif était de mesurer mensuellement, in situ, dans la subéraie, les vitesses d'ingestion des herbacées, des arbustes non fixateurs d'azote et de T. linifolia par des ovins et des bovins.

\section{Site expérimental}

Les mesures de comportement alimentaire ont eu lieu de juin 1997 à mai 1998 dans la partie occidentale de la forêt de la Mâamora, de bioclimat subhumide à hiver chaud.

Le site expérimental est représentatif du climax de la Mâamora, l'association à T. linifolia et Quercus suber, sur sol profond de 1 à $2 \mathrm{~m}$ ou davantage, de sable sur argile, favorable à un bon développement des ligneux.

La strate arborée est représentée par le chêne-liège, Q. suber (L.); la strate arbustive par T. linifolia (L.) Webb et Berth, et par des arbustes non fixateurs; trois sont

\section{Tableau 2. Évolution de la population humaine, de la fréquentation du bétail et de la couverture forestière (1960-2004).}

Table 2. Evolution of inhabitants, sheep and cattle numbers and forest area evolution (1960-2004).

\begin{tabular}{|c|c|c|c|}
\hline & 1960 & 1993 & 2004 \\
\hline Population urbaine ${ }^{a}$ & $100 \%$ & $156 \%$ (environ $3000000^{b}$ ) & $412 \%$ \\
\hline Population rurale ${ }^{a}$ & $100 \%$ & $290 \%$ (environ 300 000) & $338 \%$ \\
\hline \multicolumn{4}{|l|}{ Effectifs } \\
\hline Bovins & 24000 & $52000^{b}$ & - \\
\hline Ovins & 52000 & $173000^{b}$ & - \\
\hline Couverture (hectares) & 1950 & $1998^{c}$ & $2001^{d}$ \\
\hline Chêne-liège & 102279 & 60550 & 58396 \\
\hline À densité forte & & & 4110 \\
\hline Moyenne & & & 16665 \\
\hline Faible & & & 37621 \\
\hline Eucalyptus & 30002 & 40350 & 60621 \\
\hline Pins maritimes & 1123 & 14100 & 5686 \\
\hline Acacia mollissima & & 1000 & \\
\hline Clairières & 449 & 10824 & 7350 \\
\hline
\end{tabular}

a Évolution en pourcentage au prorata de l'évolution nationale (Haut Commissariat au Plan, 2004 et Centre d'études et de recherches démographiques, 1997).

${ }^{\mathrm{b}}$ Mhirit et al., 1995.

c Benzyane, 1998.

relativement bien appétés: Lavan- rops bumilis (L.), abondant et à feuillage dula stoeckas (L.), abondant mais de persistant en été, Cistus salviifolius (L.), feuillage peu persistant en été, Chemae- peu abondant mais persistant; un

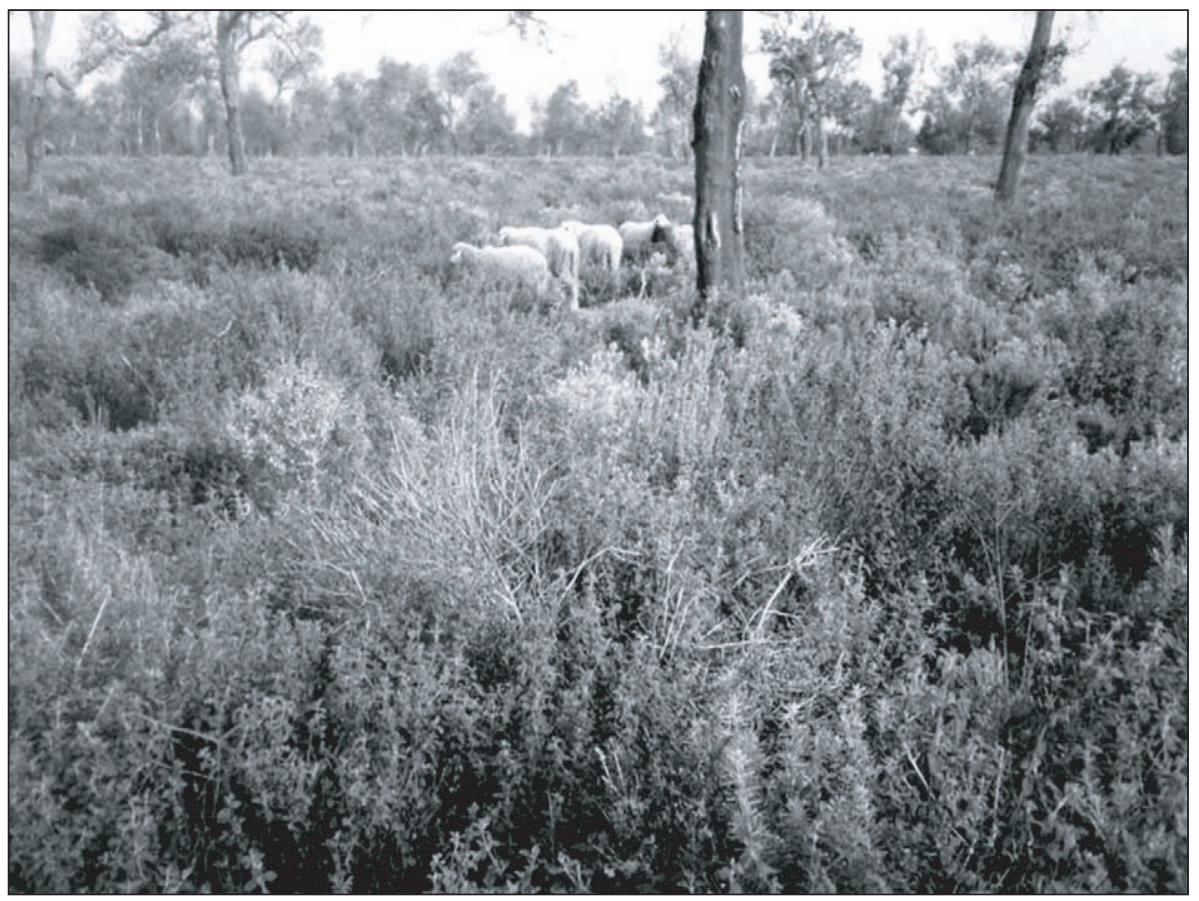

Figure 2. Ovins broutant Teline dans la subéraie de la Mâamora en hiver.

Figure 2. Sheep browsing Teline in the Maamora cork tree forest in winter time. 
quatrième, Thymelaea lythrö̈des Barr et Murbeck, n'est pas appété ; la strate herbacée est dominée par les graminées annuelles, mieux adaptées que les pérennes au surpâturage, omniprésent en Mâamora. Le recouvrement arborescent était de 50 à $60 \%$ en projection verticale, de 15 à $25 \%$ pour les arbustes non fixateurs et de 25 à $35 \%$ pour T. linifolia. Le recouvrement basal des herbacées était de 25 à $35 \%$.

\section{Choix des animaux, conduite du pâturage et suivi du comportement alimentaire}

Le comportement alimentaire d'animaux du troupeau local (figures 2,3) a été observé les 15 et 16 de chaque mois pendant 12 mois à partir de juin 1997. Conduits dans le troupeau et habitués à cette végétation dès leur jeune âge, ils pâturaient en forêt toute l'année. Quittant la bergerie dès le matin, ils y revenaient le soir après une journée entière dans la subéraie.

Les animaux ont été observés l'un après l'autre par le même observateur, au cours de séquences d'activité de pâturage de 15 minutes, le matin et l'après-midi. Un contrat moral établi avec le berger a permis de suivre les mêmes animaux sur 12 mois, en parcours gardienné, soit quatre génisses vides de moins de deux ans pesant $130 \mathrm{~kg}$ et quatre antenaises de moins d'un an pesant $35 \mathrm{~kg}$; ils étaient maintenus, sur l'initiative du berger et sous le contrôle de l'expérimentateur, sur un circuit caractéristique du type de végétation ciblé. Les nombres de coups de dent donnés par chaque animal dans les herbacées, les arbustes non fixateurs et Teline ont été comptés, l'observateur se

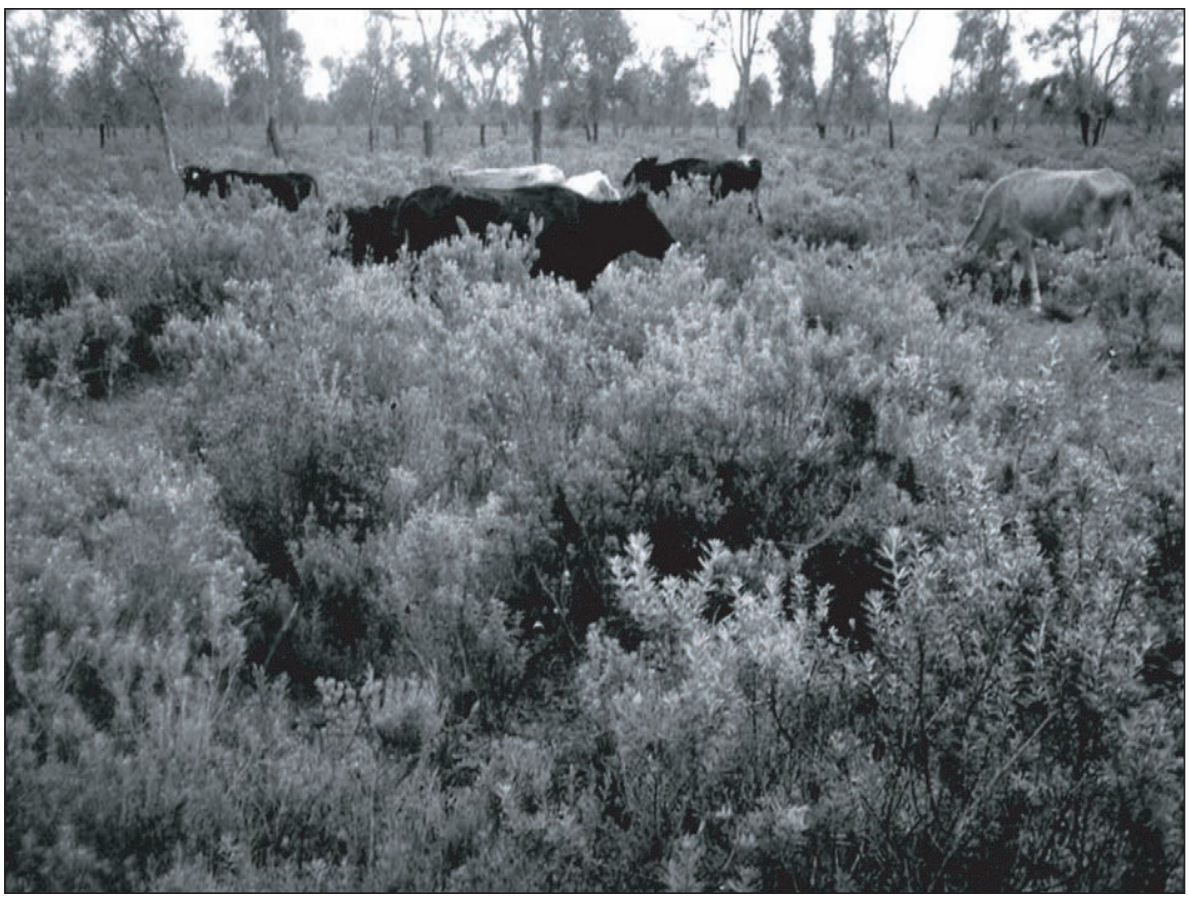

Figure 3. Bovins broutant Teline dans la subéraie de la Mâamora en hiver.

Figure 3. Cattle browsing Teline in the Maamora cork tree forest in winter time.

tenant à quelques mètres de distance, mais assez proche pour identifier la catégorie concernée à chaque coup de dent.

Une simulation du prélèvement de l'animal par prélèvement manuel (band plucking method) de 100 échantillons par catégorie a été réalisée en fin de journée de façon à estimer le poids de la bouchée (Prache et Peyraud, 1997). Ces échantillons ont été pesés après passage à l'étuve pendant 24 heures.
Ces observations ont permis de déterminer pour chaque mois et par catégorie :

- le nombre moyen de coups de dent par minute ;

- le poids sec moyen des bouchées ;

- la vitesse d'ingestion, produit du nombre de coups de dents par minute par le poids sec moyen des bouchées.

Les résultats sont exprimés en vitesse absolue d'ingestion ( $\mathrm{dg} / \mathrm{min}$ ) et en vitesse d'ingestion relative par catégorie. L'évolu-

\section{Tableau 3. Températures moyennes mensuelles, précipitations mensuelles et indice ombrothermique P/2T (sur l'intervalle entre deux séries de mesures de vitesse d'ingestion).}

Table 3. Monthly mean temperature, total rainfall and P/2T ratio (on the basis of the interval between two ingestion-rate monitoring series).

\begin{tabular}{lllllll}
\hline & Mai-juin & Juin-juillet & Juillet-août & $\begin{array}{l}\text { Août- } \\
\text { septembre }\end{array}$ & Septembre-octobre & $\begin{array}{l}\text { Octobre- } \\
\text { novembre }\end{array}$ \\
\hline Température moyenne $\left({ }^{\circ} \mathrm{C}\right)$ & 18,9 & 20,8 & 22,4 & 22,7 & 21,3 & 19,7 \\
Précipitations $(\mathrm{P}) \mathrm{mm}$ & 9 & 2 & 0 & 0 & 21,5 & 17 \\
$\mathrm{P} / 2 \mathrm{~T}$ & 0,23 & 0,05 & 0 & 0 & 0,47 & 0,43 \\
& $\begin{array}{l}\text { Novembre- } \\
\text { décembre }\end{array}$ & $\begin{array}{l}\text { Décembre- } \\
\text { janvier }\end{array}$ & $\begin{array}{l}\text { Janvier- } \\
\text { février }\end{array}$ & Février-mars & Mars-avril & Avril-mai \\
Température moyenne $\left({ }^{\circ} \mathrm{C}\right)$ & 15,2 & 14,3 & 14,9 & 15,5 & 16,6 & 16,9 \\
Précipitations mm & 206 & 116 & 119 & 0 & 37,9 & 11,4 \\
P/2T & 6,07 & 3,78 & 4,58 & 0 & 1,1 & 0,32 \\
\hline
\end{tabular}


tion des vitesses d'ingestion sur 12 mois est représentée par la courbe de tendance. Cette méthode facilite la mise en évidence de liaisons du comportement alimentaire des animaux avec l'évolution du climat mois par mois ; les données climatiques mensuelles ont été calculées sur l'intervalle d'un mois entre deux observations (tableau 3). L'état global de la végétation herbacée et des arbustes a été expertisé : croissance ou arrêt, vert ou sec, rameaux et feuilles jeunes ou matures ; la phénologie de Teline a été observée chaque mois

\section{Résultats}

Le climat est marqué par un très fort déficit hydrique 8 mois sur $12(\mathrm{P} / 2 \mathrm{~T}<1$; tableau 3). Dans ce contexte, la croissance de l'herbe, des arbustes et de Teline a été nulle jusqu'au 15 septembre; les annuelles ont commencé à germer et Teline à débourrer et à feuiller à la suite de petites pluies de fin septembre et d'octobre ; avec les grandes pluies qui débutent le 20 novembre, la croissance de l'herbe a commencé et Teline est reparti de souche et a reconstitué son feuillage ; la repousse des arbustes non fixateurs a attendu la fin de l'hiver; la croissance de l'herbe s'est intensifiée de décembre à février et a été ralentie par le déficit hydrique du début mars; en mars-avril, elle a repris un rythme modéré, et les jeunes pousses de Teline se sont lignifiées ; à partir de mi-avril, le déficit hydrique se creuse, la croissance de l'herbe est faible.

\section{Régularité de l'évolution de la vitesse d'ingestion sur l'année}

Les vitesses d'ingestion des arbustes et de Teline suivent une évolution régulière, avec certaines particularités liées à l'espèce animale. Les courbes de tendance (non montrées) rendent compte de 92 à $96 \%$ de la variance.

Les vitesses d'ingestion des herbacées sont plus irrégulières (figures 4, 5), les courbes de tendance rendant compte de $76 \%$ de la variance. Eu égard à l'évolution très régulière observée sur les arbustes et Teline, l'hypothèse la plus probable est une évolution régulière sur l'herbe aussi, masquée par la variabilité des mesures en temps bref sur une strate herbacée de répartition hétérogène. Dans cette hypothèse, les valeurs de la vitesse d'ingestion

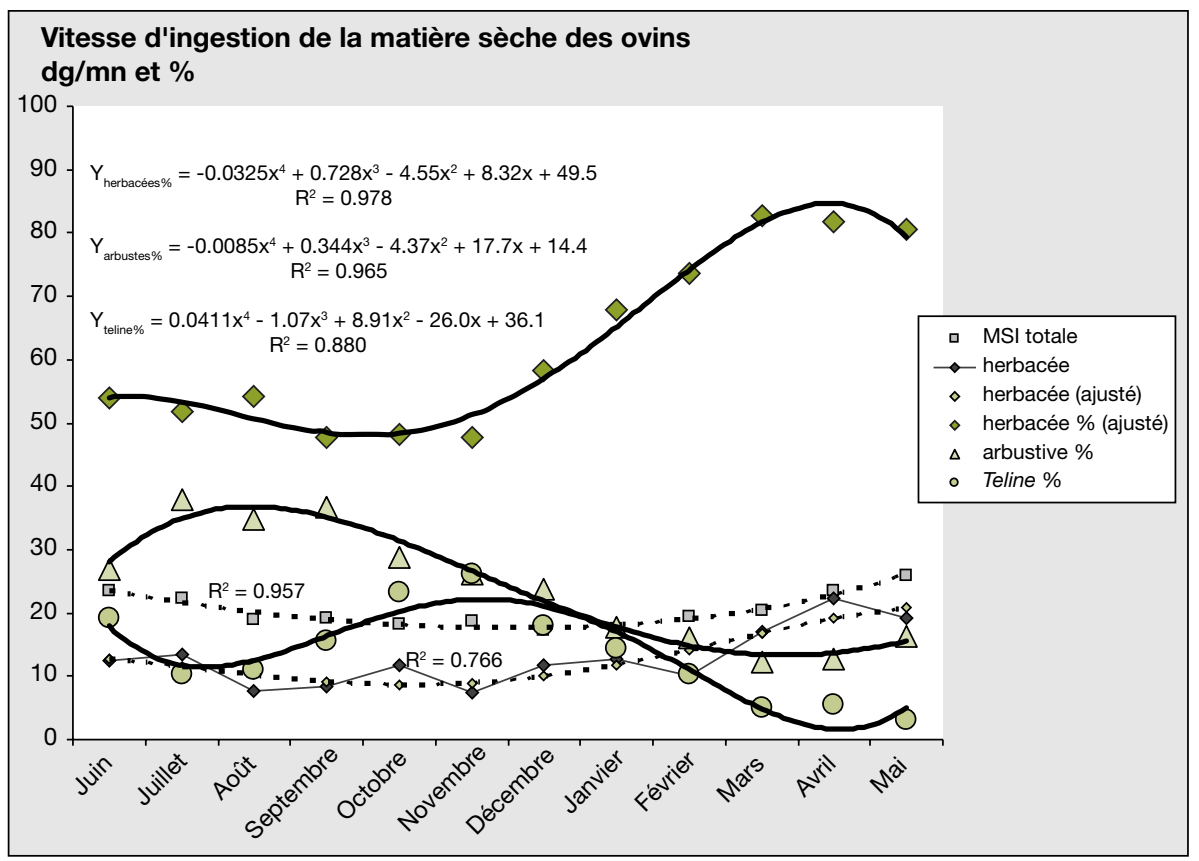

Figure 4. Ovins : vitesse d'ingestion de la matière sèche.

Figure 4. Sheep: dry matter ingestion rate.

: contribution en pour cent des herbacées, des arbustes et de Teline ;

........ : MS totale et MS herbacée (dg/min).

MS : matière sèche; $\mathrm{MSI}$ : matière sèche ingérée.

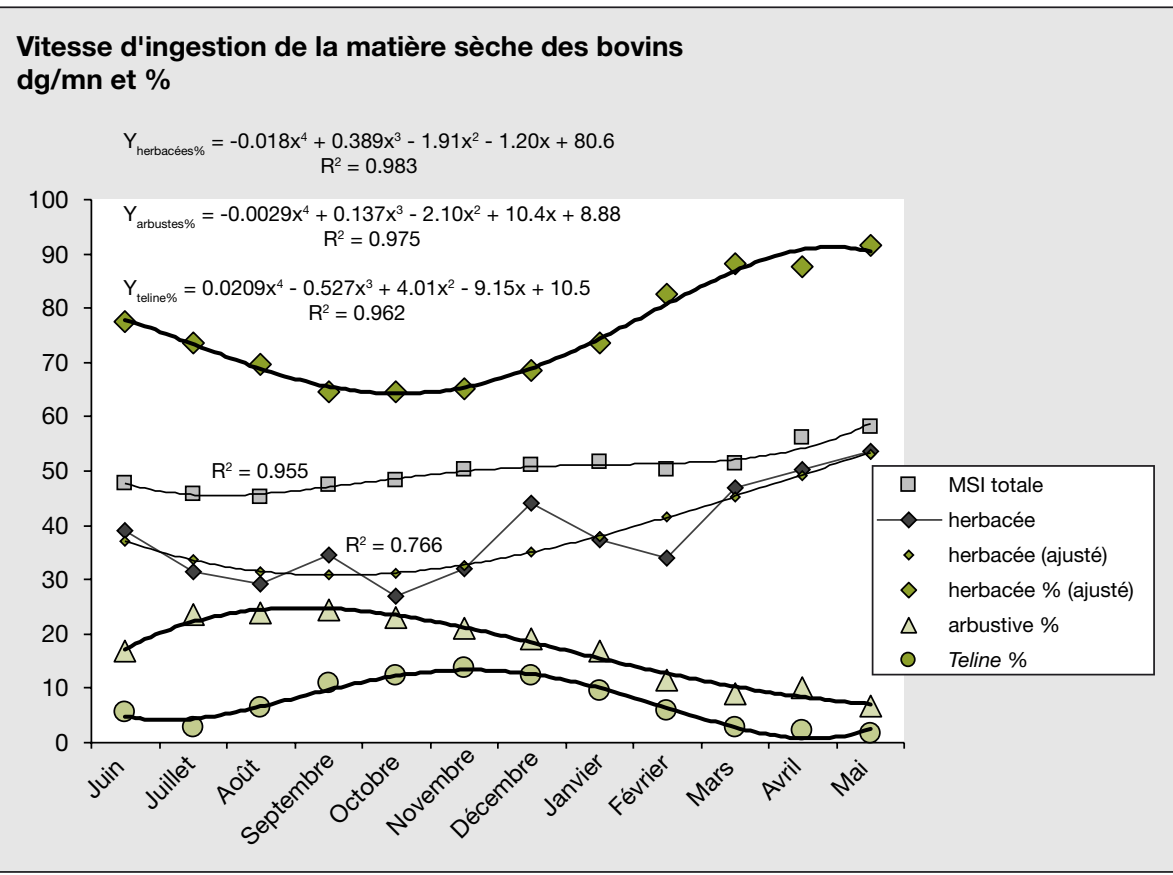

Figure 5. Bovins : vitesse d'ingestion de la matière sèche.

Figure 5. Cattle: dry matter ingestion rate.

: contribution en pour cent des herbacées, des arbustes et de Teline; : MS totale et MS herbacée ( $\mathrm{dg} / \mathrm{min}$ ).

MS : matière sèche; MSI : matière sèche ingérée. 
des herbacées ont été ajustées à leur courbe de tendance pour le calcul de la vitesse d'ingestion globale.

\section{Vitesses d'ingestion des ovins et climat}

En période sèche ou de faibles pluies, l'herbe est sèche; les ovins limitent la contribution des herbacées à $50 \%$; ils reportent leur choix sur les feuilles et jeunes rameaux des arbustes non fixateurs ( $27 \%$ en juin, $35 \%$ de juillet à septembre, $29 \%$ en octobre); Teline est bien consommé en juin (19\%) pour ses gousses, et lorsqu'il renouvelle ses organes verts en octobre (23\%) et novembre (26\%), alors que les feuillages des autres arbustes vieillissent et s'éclaircissent. (figure 4 ; tableau 3).

Les substitutions sont imparfaites: la vitesse d'ingestion globale décroît de 24 à $19 \mathrm{dg} / \mathrm{min}$ de juin à août $(-26 \%)$ et stagne d'août à janvier.

Après les fortes pluies de novembre à janvier, la vitesse relative d'ingestion des herbacées évolue de $50 \%$ en novembre à $80 \%$ de mars à mai, en parallèle à l'offre croissante d'herbe nouvelle. Celle des arbustes décroît de novembre (26\%) à avril $(13 \%)$ et reprend en mai $(16 \%)$ à la maturation de l'herbe. La contribution de Teline décroit considérablement de décembre (18\%) à mai (3\%).

La vitesse d'ingestion globale croît à nouveau avec l'accumulation d'herbe jeune de janvier à mai où elle atteint $26 \mathrm{dg} /$ min. En période sèche, la collecte insistante des ovins sur les arbustes a contribué à ralentir leur ingestion.

\section{Vitesses d'ingestion des bovins et climat}

Dans la période de déficit hydrique, les vitesses relatives d'ingestion des herbacées par les bovins diminuent de juin $(78 \%)$ à octobre et novembre $(65 \%)$. Les bovins équilibrent leur ration avec les organes verts des arbustes: assez bien appétés en juin (17\%), leur contribution est stable et importante (24\%) de juillet à octobre, voire à novembre (21\%); Teline est peu appété en juin $(6 \%)$, très peu en juillet $(3 \%)$; sa contribution augmente régulièrement jusqu'à un palier modeste $(12 \%)$ de septembre à décembre (figure 5 ; tableau 3).

Les substitutions fonctionnent bien: la vitesse globale d'ingestion est soutenue en été avec un palier inférieur peu marqué ( $45 \mathrm{dg} / \mathrm{min}$ ) en juillet et août

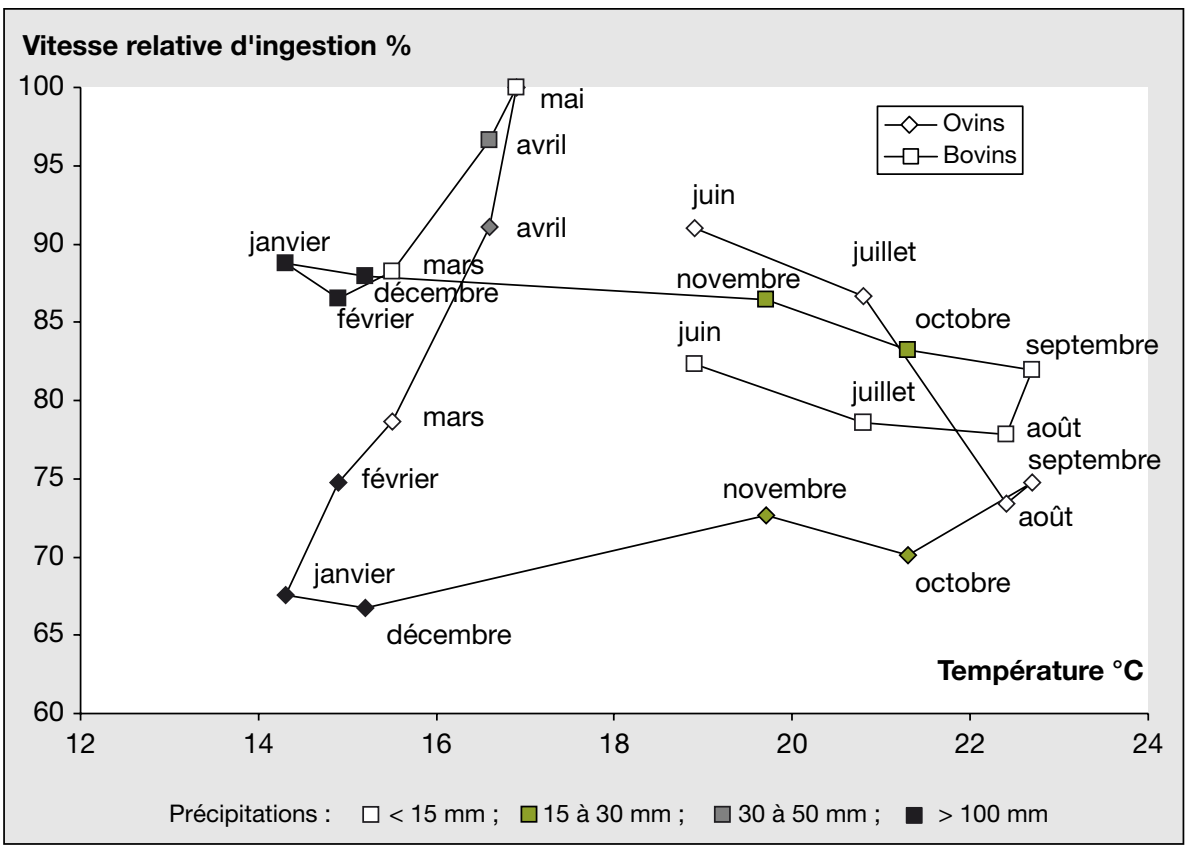

Figure 6. Vitesse relative d'ingestion de la matière sèche par rapport à la vitesse d'ingestion maximale (observée en mai) : relation avec la température moyenne et les précipitations mensuelles.

Figure 6. Relative dry matter (DM) ingestion rate to maximum rate (May): relationships with monthly mean temperatures and rainfall.

Le mot " septembre " désigne la période du 16 août au 15 septembre en conformité avec les dates d'observation du comportement alimentaire; il en va de même pour les mois suivants et dans les Figures 8 et 9.

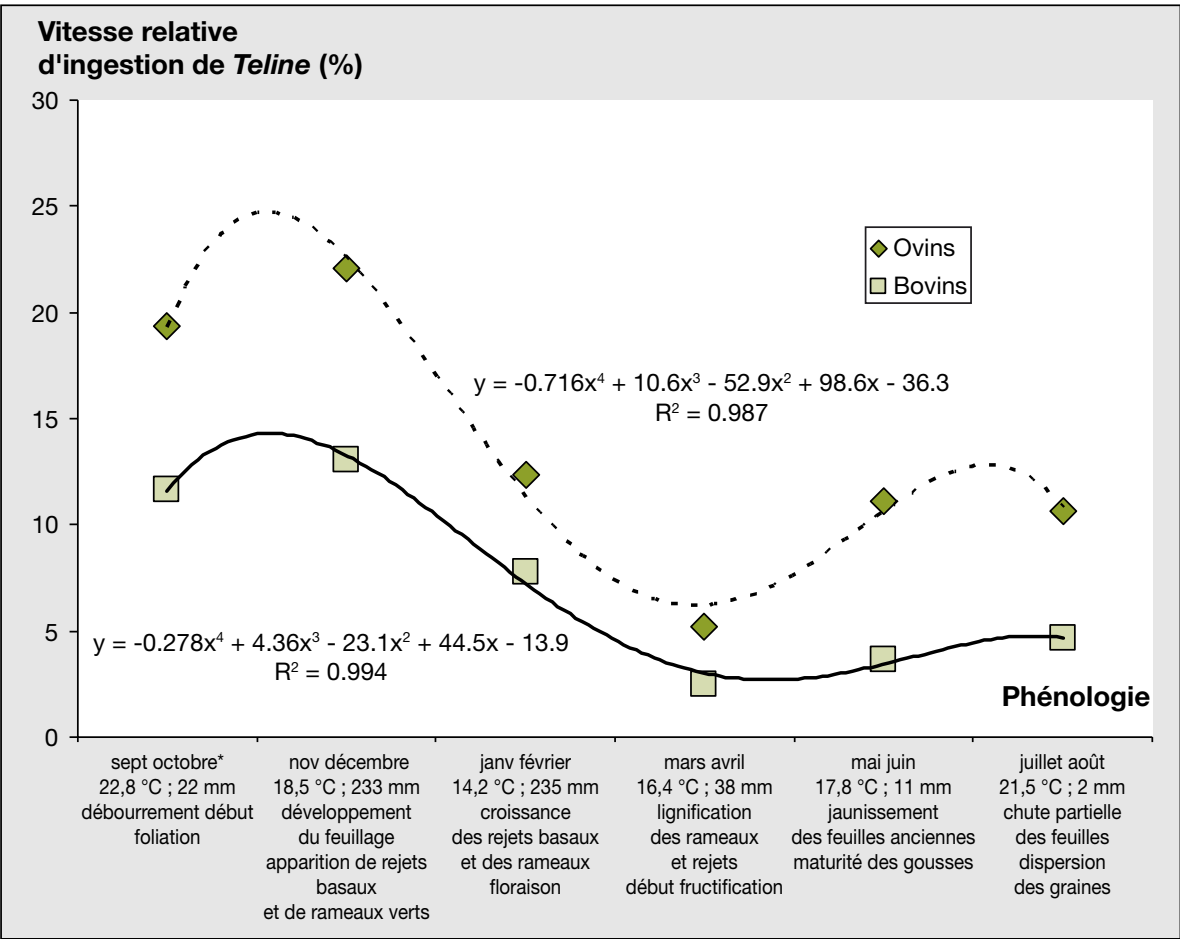

Figure 7. Vitesse relative d'ingestion de Teline (en pour cent de la vitesse d'ingestion globale) en relation avec la température moyenne, le cumul des précipitations et la phénologie de l'espèce.

Figure 7. Teline relative ingestion rate (percent of total ingestion rate) as related to mean temperatures, total rain and phenology.

*L'expression "sept octobre " couvre la période du 16 août au 15 octobre pour correspondre aux dates d'observation des comportements alimentaires. 
(soit $-5 \%$ par rapport à juin) où l'herbe est sèche mais encore abondante; elle croît jusqu'à $50 \mathrm{dg} / \mathrm{min}$ en automne avec les temps plus frais.

Après le retour des pluies, la contribution des herbacées croît fortement, de novembre (65\%) à mai (92\%). Celle des arbustes, moins attrayants que l'herbe jeune, décroît fortement : de $21 \%$ en novembre à $7 \%$ en mai. Celle de Teline décroît régulièrement à partir de janvier (9\%) jusqu'en avril et mai (2\%).

En prélevant préférentiellement l'herbe en toute saison, les bovins ont maintenu leur rythme d'ingestion, même en été ; ils l'ont accru fortement et ont marqué un désintérêt croissant pour les arbustes et Teline au fur et à mesure qu'augmentait l'offre d'herbe verte.

\section{Discussion}

Les herbivores sur parcours modulent leur ration selon la disponibilité et l'appétibilité des espèces (Meuret, 2005 ; Roguet et al., 1998). Ils adaptent leur comportement alimentaire en fonction des changements saisonniers de la végétation pour maintenir leur niveau d'ingestion (Agreil et al., 2005).

Les vitesses d'ingestion reflètent les états de la végétation à travers le prisme du comportement alimentaire spécifique des ovins et des bovins. Prenant comme indice 100, la vitesse d'ingestion maximale, observée en mai, trois périodes sont identifiables dans l'évolution comparée des vitesses d'ingestion globale (figure 6) :

- de juin à août, une période de sécheresse et de températures élevées avec diminution des ressources et de leur appétence ; - de septembre à janvier, une période de difficulté croissante pour les ovins pénalisés par leur attirance pour les arbustes et le tri fin, et de difficulté décroissante pour les bovins par leur aptitude à maintenir une forte contribution des herbacées et de larges bouchées ;

- de janvier à mai, une accélération de l'ingestion des herbacées convergeant en mai entre bovins et ovins au fur et à mesure de l'augmentation de l'offre de matériel vert, reconstitué après le retour des grandes pluies d'hiver et sous la montée des températures printanières.

La croissance de Teline d'octobre à décembre, alors que les herbacées et les arbustes non fixateurs sont vieillis ou peu productifs, rend compte du report partiel du choix des animaux sur cette espèce à cette période (figure 7). Par sa phénologie particulière, Teline joue un rôle clé dans l'alimentation des troupeaux durant l'automne.

Les différences de vitesse d'ingestion relativement à celles de mai entre ovins et bovins résultent de différences au niveau du rythme de préhension (nombre de coups de dent par minute) et du poids relatif des bouchées.

\section{Rythme de préhension}

Chez les deux espèces, le rythme de préhension décroît lorsque la température croît (figure 8) ; en période de fortes températures (juin à novembre), il est plus fortement ralenti en jours longs qu'en jours courts (températures maximales plus élevées en jours longs). Ovins et bovins ont un rythme voisin en période d'abondance (juin et juillet 1997 ; avril et mai 1998); les ovins sont significativement moins rapides (8 mois sur 12), d'août à mars, c'est-à-dire après accentuation de la sécheresse et avant la pleine croissance de l'herbe, dans une période où leur activité de tri sur les arbustes est la plus grande.

\section{Poids des bouchées}

Pour comparer les deux espèces, le poids moyen des bouchées a été converti en poids moyen relatif des bouchées (pmrb) (pour cent du poids des bouchées de mai, période d'offre abondante ; figures 4,5); le pmrb croît linéairement avec la température (figure 9); la pente est plus faible en jours courts (de septembre à mars) qu'en jours longs (mars à août) en rapport avec l'intensité de l'évapotranspiration et la dessiccation du matériel végétal prélevé. En saison humide (octobre à avril), le pmrb des ovins est inférieur à celui des bovins, suggérant qu'ils opèrent un tri fin d'organes plus tendres.

La taille de la mâchoire et le mode de préhension seraient déterminants dans les choix alimentaires (Prache et Peyraud, 1997) : la mâchoire étroite et le pincement des organes végétaux permettent aux ovins d'effectuer un tri fin et facilitent

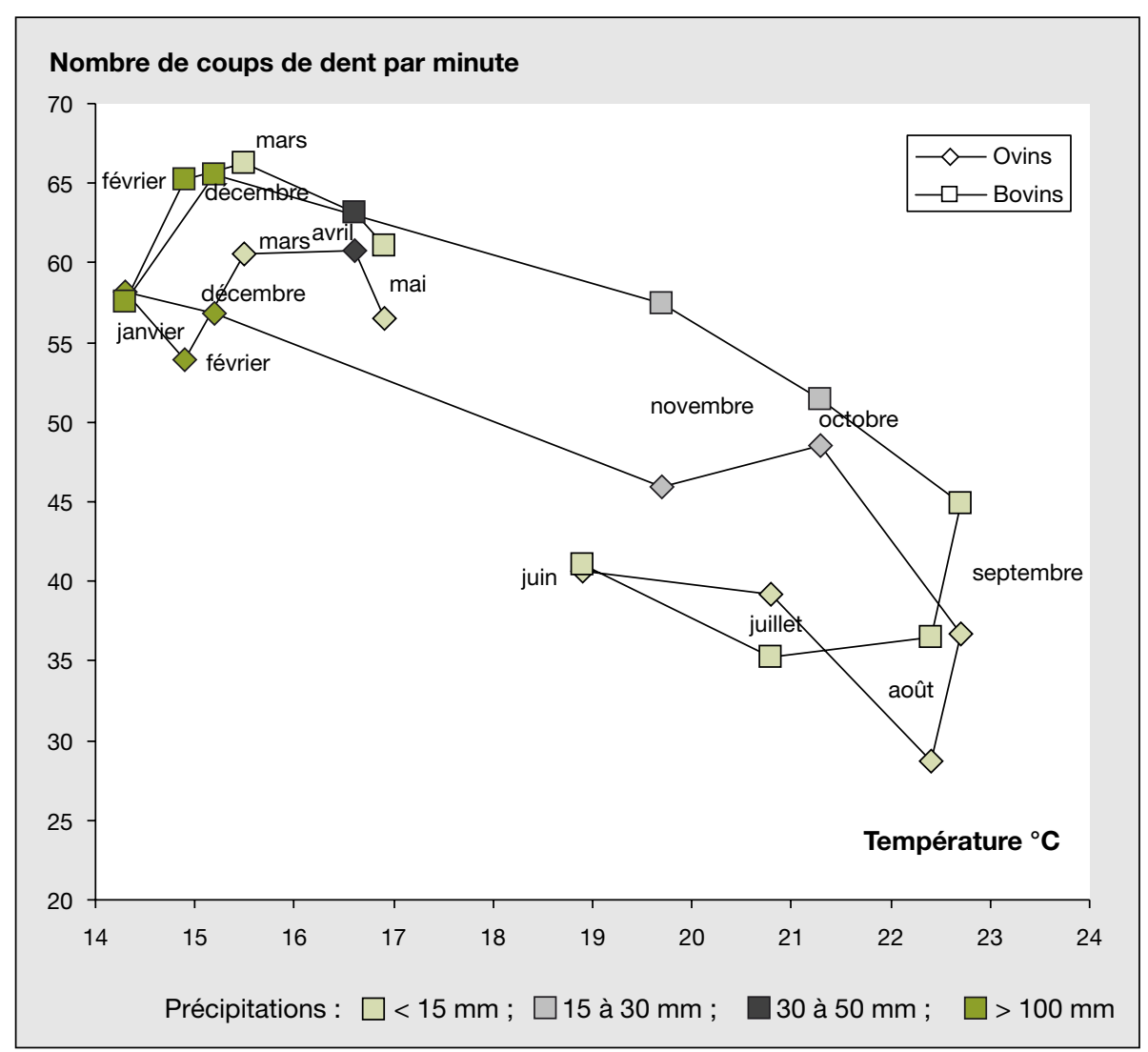

Figure 8. Nombre de coups de dent par minute : relation avec la température moyenne et les précipitations mensuelles.

Figure 8. Number of bites/min: relationships with monthly mean temperatures and rainfall. 


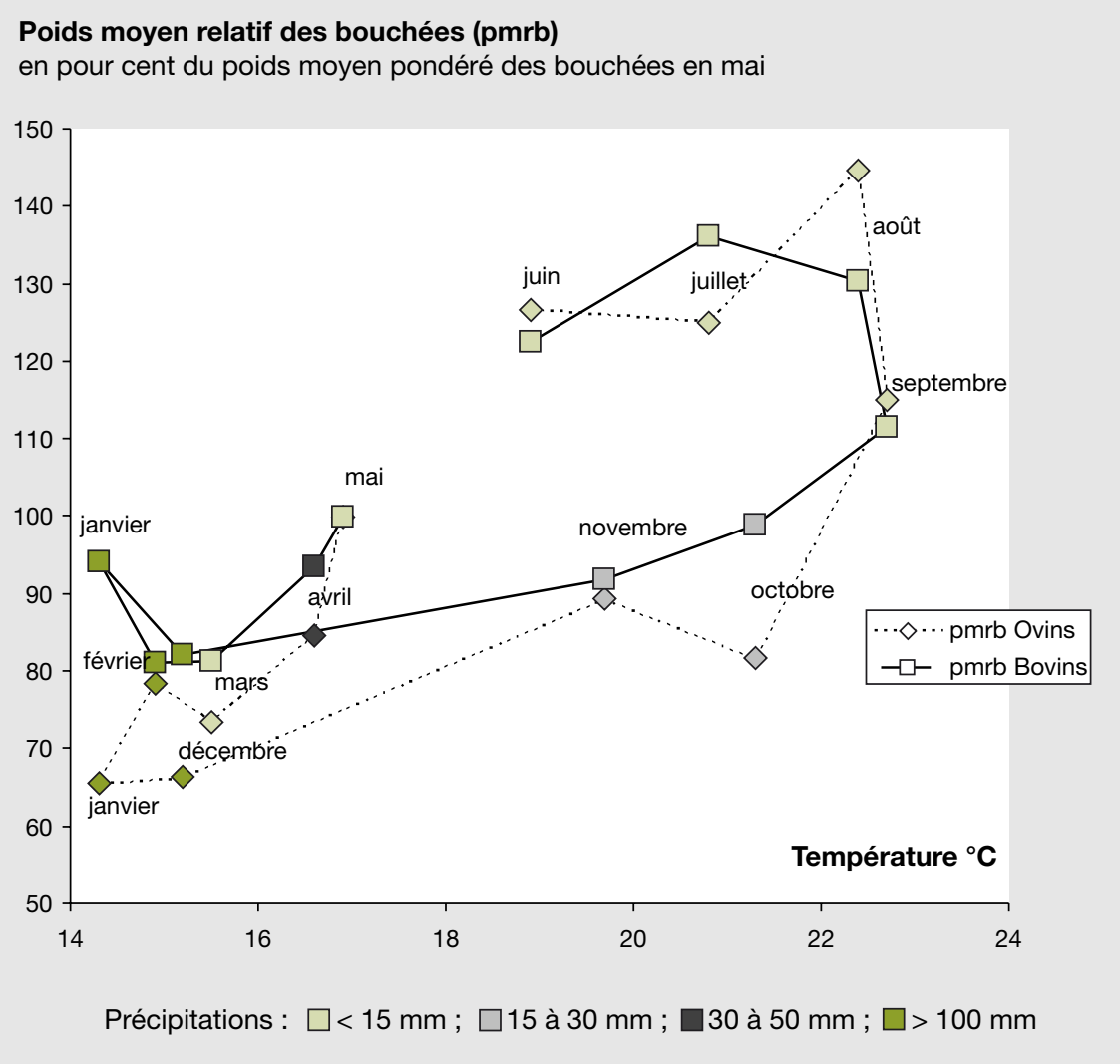

Figure 9. Poids sec moyen relatif des bouchées : relation avec la température moyenne et les précipitations mensuelles.

Figure 9. DM mean relative weight per bite: relationships with monthly mean temperatures and rainfall.

leur report de l'herbe desséchée vers les organes verts arbustifs. La mâchoire large et la préhension des organes végétaux par la langue des bovins, leur rend moins aisé le tri des organes verts des arbustes et plus facile la préhension de larges bouchées d'herbe, qu'elle soit desséchée ou verte.

\section{Place de Teline dans l'aménagement général de la subéraie}

Les recommandations des deuxièmes assises de la recherche forestière (Centre de recherche forestière, 2008) soulignent l'intérêt de "l'approche intégrée, spatialisée et partenariale adoptée pour la mise en ouuvre du plan de sauvegarde et de réhabilitation de la Mâamora (permettant) de concilier la pérennisation de cette forêt et les besoins de la population".

La transition démographique étant largement accomplie, il devient plus aisé de réguler la gestion de la forêt sur la base d'un recensement exhaustif des usagers et d'une typologie des systèmes d'élevage
(Naggar, 1995), installés ou visiteurs périodiques, et de concevoir une intégration des fonctions multiples de Teline (améliorateur des sols, combustible domestique, ressource pastorale et apicole) dans un plan d'aménagement et de rajeunissement de la subéraie (Guérin et Macron, 2005).

Teline constitue une importante ressource pastorale d'octobre à février et contribue ainsi à limiter les ébranchages intempestifs. Mais pour limiter drastiquement ces dégâts, les ressources du parcours sont insuffisantes dès mi-juillet jusqu'en fin d'hiver pour les ovins, et de juin à octobre pour les bovins (figure 6). De juillet à mars, les vitesses d'ingestion marquent des déficits de $28,6 \%$ chez les ovins et $14,9 \%$ chez les bovins par rapport à la période d'abondance de mai.

D'après la carte d'Aafi (2007), la zone étudiée est peu dégradée ; sur l'ensemble de la subéraie, la densité du couvert et la qualité de la végétation sont nettement inférieures, et les déficits plus à craindre. L'allègement du chargement en bétail est une nécessité, notamment en retirant assez tôt les jeunes animaux destinés à l'engraissement en début d'été (Qarro et al., 2000). L'ébranchage illégal doit être remplacé par un élagage d'entretien favorisant la glandée, réalisé par des ouvriers compétents. Le complément principal pour le troupeau reproducteur vivant en forêt pourrait être produit en culture sèche du trèfle souterrain, intercalaire dans les subéraies les moins denses (Masson, 1995) et dans les parcelles dépourvues d'arbres; les analyses foliaires de Teline du site étudié ont montré une nutrition phosphatée correcte et un très fort déficit potassique (Hracherrass, 1993); un diagnostic de printemps sur l'herbe et les feuillages, par type de milieu, révèlera le niveau nutritionnel ; une fertilisation modérée en $\mathrm{P}$ ou en $\mathrm{K}$ peut se révéler indispensable à la réussite en cas de carence drastique. En période sèche, une rotation de pâturage entre le parcours, la culture agroforestière et les ressources hors forêt dépend d'une organisation sociale cohérente des usagers.

Le rajeunissement de la subéraie (Belghazi et al., 2001) nécessite la protection des semis en place ou la plantation de plants produits en pépinière. La technique de production de plants à fort chevelu de racines et faible développement aérien permet un taux de reprise élevé. Pour accélérer l'installation, une expérimentation utile serait de combiner l'ameublissement du sol en profondeur jusqu'à $150 \mathrm{~cm}$ sur la ligne de plantation et la mise en défens (clôtures) ou la protection individuelle (tubes ou filets) des plants avec semis en ligne de Teline à $50 \mathrm{~cm}$ de part et d'autre et sous-solage à $80 \mathrm{~cm}$ sous le semis. L'effet protecteur principal de Teline est l'ambiance climatique créée progressivement jusqu'à une hauteur de $1,5 \mathrm{~m}$ autour du pied du jeune arbre; l'étude du comportement hydrique (Balandier et al., 2008) des jeunes chênes-lièges dans un entourage de Teline, à découvert ou en subéraie dégradée, voire dans des plantations de pins ou d'eucalyptus fortement éclaircies en vue de leur conversion, en permettrait la validation.

\section{Conclusion}

Le maintien de peuplements de Teline jeunes et productifs en organes verts est totalement justifié du point de vue pasto- 
ral. Il l'est aussi pour le redéploiement de la subéraie en raison de la protection microclimatique qu'il exercerait sur les jeunes chênes-lièges et de sa fixation symbiotique de l'azote estimée à $50 \%$ du contenu azoté de la plante, soit 35 à $120 \mathrm{~kg} /$ ha selon la profondeur du sol et la densité du couvert arborescent (Hracherrass, 1993 et 2004). Récolté avant la sénescence du peuplement, Teline constitue une ressource de bois de feu équivalente à $3160 \mathrm{~L} /$ ha de fuel pour $7 \mathrm{t} / \mathrm{ha}$ de biomasse sèche (Hracherrass, 1993).

Sous condition d'assurer une nutrition phosphatée et potassique suffisante, Teline contribuera puissamment à la régénération d'une subéraie productive en liège, de ses ressources pastorales, apicoles et énergétiques et de sa biodiversité.

\section{Références}

Aafi A. Diversité floristique de l'écosystème à chêne-liège de la Mâamora. Thèse IAV HassanII, Rabat, 2007.

Agreil C, Fritz H, Meuret M. Maintenance of daily intake through bite mass diversity adjustment in sheep grazing on heterogeneous vegetation. Appl Anim Behav Sci 2005 ; 91 : 35-56.

Balandier P, De Montard FX, Curt T. Root competition for water between tree and grass in a silvopastoral plot of 10-year-old Prunus avium. In : Batish DR, Kohli RK, Jose S, Singh HP, eds. Ecological basis of agroforestry. London : CRC, 2008.

Belghazi B, Ezzahiri M, Amhajar M, Benzyane $M$. Régénération artificielle du chêne-liège en forêt de la Mâamora (Maroc). Forêt méditerranéenne $2001 ; 13$ : 253-61.
Benzyane M. La subéraie marocaine, produit économique et social à développer. In : La régénération des forêts de chêne-liège. Tunis: INRGREF, 1998.

Benzyane M, Naggar M, Lahlou B. L'aménagement des forêts sud-méditerranéennes : quelle approche? Forêt méditerranéenne 2002; 23 : 201-10.

Berkat O. Évaluation des ressources pastorales dans la forêt de la Mâamora: appui scientifique aux aménagements sylvopastoraux. In : Rejdali M, ed. Gestion des ressources pastorales. Rabat: IAV Hassan-II, 1995.

Centre d'études et de recherches démographiques (Cered). Situation et perspectives démographiques du Maroc. Rabat: Haut Commissariat au Plan, 1997.

Centre de recherche forestière (CRF). Réhabilitation des subéraies. Actes des deuxièmes assises de la recherche forestière, mai 2007. Kénitra (Maroc) : CRF, 2008.

Fraval A, Villemant C. La Mâamora et ses ennemis. Inra les dossiers de l'environnement 1997 ; $15: 133-46$.

Guérin G, Macron MC. Sylvopastoralisme. Les clés de la réussite. Paris: Institut de l'élevage, 2005

Hracherrass A. Étude des peuplements de Teline linifolia en forêt de la Mâamora. Thèse, université d'Aix-Marseille-III, Marseille, 1993.

Hracherrass A. Comportement écopédologique et intérêt pastoral de Teline Linifolia dans l'écosystème à chêne-liège et à $T$. Linifolia en forêt de la Mâamora. Thèse IAV Hassan-II, Rabat, 2004

Iraki A, Souafi M, Tamim M. Évaluation et révision du plan d'aménagement de la forêt de la Maâmora phase I. Projet: CCP/MOR/010/DEN SI: sn, 1991.

Masson P. Influence of silvopastoral management on the functioning of the cork-oak forest. Options Méditerranéennes 1995 ; 12 : 175-8.
Mejjati Alami M. The impact of grazing on forage quantity, quality and foliar cover of the herbaceous vegetation in the Maamora cork oak forest. Thèse, IAV Hassan-II, Rabat 1991.

Meuret M. Ressources de parcours pour ruminants. In : Duplan JM, ed. Manuel de zootechnie comparée. Paris: Inra éditions, 2005.

M'hirit O, Benzyane M, Naggar M. Aménagement sylvopastoral: un outil de gestion et un préalable à la sauvegarde des écosystèmes forestiers marocains. ARF Atelier sylvopastoralisme. Salé (Maroc) : Enfi, 1995.

Nafaa R. Dynamique du milieu naturel de la Mâamora et de ses bordures. Paléoenvironnements et dynamique actuelle. Thèse université Mohammed V, Rabat, 1997.

Naggar M. Problématique d'aménagement sylvopastoral au Maroc. Cas de la forêt de la Mâamora. Options Méditerranéennes 1995; 12: 165-8.

Prache S, Peyraud JL. Préhensibilité de I'herbe chez les bovins et les ovins. INRA Prod Anim $1997 ; 10: 377-90$

Qarro M. Effet des couverts arboré et arbustifs sur les potentialités herbagères en forêt de la Mâamora occidentale (Maroc). Options Méditerranéennes $1995 ; 12$ : 103-6.

Qarro M, Berkat O, M'Hamedi M, Yessef M. $L^{\prime}$ aménagement sylvopastoral : contraintes et développement durable. In: Bourbouze $A$, Qarro M, eds. Rupture : nouveaux enjeux, nouvelles fonctions, nouvelle image de l'élevage sur parcours. Montpellier: CIHEAM-IAMM, 2000.

Haut Commissariat au Plan (HCP). Pauvreté, développement humain et social au Maroc. Recensement général de la population et de I'habitat (RGHP). Rabat: Centre national de documentation, 2004 (1994) (cédérom).

Roguet C, Dumont B, Prache S. Sélection et utilisation des ressources fourragères par les herbivores. INRA Prod Anim 1998; 11: 273-84. 